\title{
Narrow-band and tunable intense terahertz pulses for mode-selective coherent phonon excitation $\odot$
}

Cite as: Appl. Phys. Lett. 117, 101101 (2020); doi: 10.1063/5.0015612

Submitted: 29 May 2020 . Accepted: 26 July 2020 .

Published Online: 8 September 2020

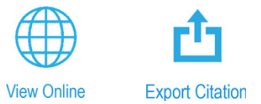

C. Vicario,' (D) A. Trisorio, ' (D) S. Allenspach, ${ }^{1,2}$ C. Rüegg, ${ }^{1,2,3,4}$ and F. Giorgianni ${ }^{1, \text { a) }}$ (D)

\author{
AFFILIATIONS \\ 'Paul Scherrer Institute, 5232 Villigen-PSI, Switzerland \\ ${ }^{2}$ Department of Quantum Matter Physics, University of Geneva, CH-1211 Geneva 4, Switzerland \\ ${ }^{3}$ Department of Physics, ETH Zurich, $\mathrm{CH}-8093$ Zürich, Switzerland \\ ${ }^{4}$ Institute of Physics, EPF Lausanne, $\mathrm{CH}-1015$ Lausanne, Switzerland
}

${ }^{\text {a) }}$ Author to whom correspondence should be addressed: flavio.giorgianni@psi.ch

\begin{abstract}
We generate frequency-tunable narrow-band intense fields in the terahertz $(\mathrm{THz})$ range by optical rectification of a temporally modulated near-infrared laser pumping a nonlinear organic crystal. Carrier-frequency tunability between 0.5 and $6.5 \mathrm{THz}$ is achieved by changing the modulation period of the laser pump. This tunable narrow-band $\mathrm{THz}$ source allows the selective coherent excitation of adjacent vibrational modes, which are demonstrated for two phonons with a frequency offset of $0.8 \mathrm{THz}$ in single-crystal $\mathrm{SrCu}_{2}\left(\mathrm{BO}_{3}\right)_{2}$. Our compact and scalable source enables an effective approach for the advanced manipulation of low-energy collective modes in condensed matter and has the potential to reveal the coupling of specific lattice vibrations with other degrees of freedom.
\end{abstract}

Published under license by AIP Publishing. https://doi.org/10.1063/5.0015612

The generation of coherent phonons using light is an emerging tool to explore the dynamics of collective lattice vibrations, and it is expected to play a major role in the ultrafast control of functional electronic, magnetic, and structural properties of solids. ${ }^{1-4}$ In conventional coherent phonon spectroscopy, sub-picosecond visible or nearinfrared lasers trigger coherent lattice displacements via Raman scattering. ${ }^{5}$ In this second-order process, which relies on excitation of the electronic system by high-energy photons, ${ }^{6,7}$ the amplitude of the coherent phonons is limited by the magnitude of the electron-lattice interaction. Moreover, the laser-driven generation of coherent phonons involves an excess of photo-induced hot electrons and, therefore, offers a restricted scalability associated with the large increase in sample temperature and ultimately with the laser-induced damage.

An efficient approach to overcome these limitations and to access phonon-mediated dynamics is based on the resonant excitation of infrared-active phonon modes using low-frequency light pulses. This radiation has the potential to generate the large-amplitude lattice displacements required to explore phonon-driven processes. Because the optical phonon frequencies in solids lie primarily in the spectral region between 1 and $10 \mathrm{THz}$, these experiments demand intense and phase-stable optical pulses at terahertz $(\mathrm{THz})$ frequencies. ${ }^{8,9}$ The recent development of strong-field quasi single-cycle $\mathrm{THz}$ sources has enabled the investigation of phonon-driven phenomena and numerous other coherent low-energy excitations, which were previously restricted. However, broadband $\mathrm{THz}$ sources drive multi-mode resonances, hindering the deep understanding of transient dynamics. An intense and narrow-band $\mathrm{THz}$ source tunable to the specific phonon resonance would greatly expand the understanding of phonon-mediated dynamics targeting distinct modes.

In this Letter, we present a scheme to generate intense narrow-band $\mathrm{THz}$ pulses by optical rectification of temporally shaped near-infrared laser pulses in a nonlinear organic salt DSTMS (4-N, N-dimethylamino- $4^{\prime}-\mathrm{N}^{\prime}$ methyl-stilbazolium 2,4,6-trimethylbenzenesulfonate). ${ }^{10}$ We demonstrate the generation of narrow-band $\mathrm{THz}$ pulses with a bandwidth less than $1 \mathrm{THz}$ and carrier frequency tunability between $0.5 \mathrm{THz}$ and $6.5 \mathrm{THz}$. The continuous tunability of intense $\mathrm{THz}$ pulses represents a key development for selective phonon excitation targeting distinct infrared-active modes. As an example, we demonstrate the selective excitation of the adjacent phonon lines in single crystal $\mathrm{SrCu}_{2}\left(\mathrm{BO}_{3}\right)_{2}$.

The scheme we present has numerous advantages such as high fields and, moreover, fills the spectral gap of the existing laser-based narrow-band $\mathrm{THz}$ sources, such as the difference-frequency generation (DFG) in organic crystals, ${ }^{11}$ which is suitable for higher frequencies $(4-18 \mathrm{THz})$, or optical rectification by temporal modulated pulses in $\mathrm{LiNbO}_{3}{ }^{12,13}$ and HMQ-TMS, ${ }^{14}$ which can cover lower frequencies of $0.3-1.3 \mathrm{THz}$ and $0.3-0.8 \mathrm{THz}$, respectively. Our source is tunable 
over a spectral range, $0.5-6.5 \mathrm{THz}$, comparable with the one demonstrated using $10 \mathrm{~Hz}$ Cr:forsterite to pump an organic crystal, ${ }^{15,16}$ but relies on a more commercially available optical-parametric amplifier (OPA) pump, which has the advantages of a higher repetition rate and a tunable wavelength for optimal pumping of different organic crystals. The higher repetition rate achievable with an OPA and the high field demonstrated here turn into a higher signal-to-noise ratio, which is crucial for future spectroscopic applications.

In the experiment, ultrashort near-infrared (NIR) laser pulses at a wavelength of $1505 \mathrm{~nm}$, energy up to $2.6 \mathrm{~mJ}$, and a pulse duration of approximately $60 \mathrm{fs}$ are generated in a three-stage OPA (Light Conversion) driven by a terawatt-class Ti:sapphire laser front end, ${ }^{17}$ shown in Fig. 1(a). The OPA pulses are temporally stretched by two pairs of high-efficiency transmission gratings ( 996.4 grooves/mm) so that the instantaneous laser frequency is chirped linearly in time across the pulse duration. The distance between the first and the second grating pair is $d=1.75 \mathrm{~mm}$. The temporal modulation is achieved by splitting, delaying, and recombining two chirped replicas in a Mach-Zehnder interferometer (MZI). For a linearly chirped optical pulse with a Gaussian envelope, ${ }^{18}$ the electric field can be described as

(a)

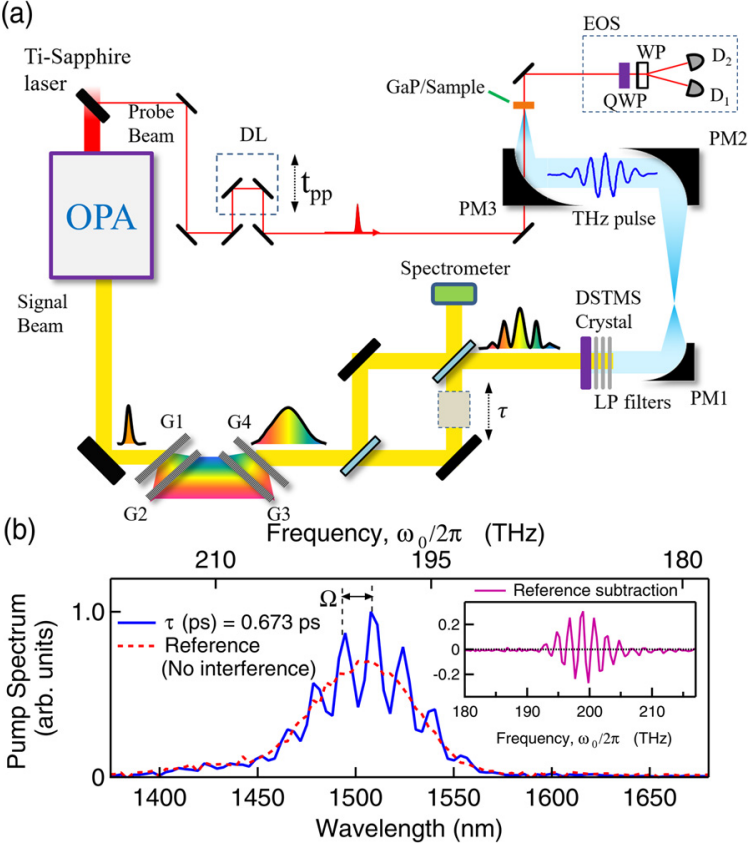

FIG. 1. Narrow-band THz generation setup. The output of an optical parametric amplifier (OPA) driven by a Ti:sapphire laser is chirped linearly by four highefficiency transmission gratings (G1-G4). The pulses are split and delayed in the Mach-Zehnder interferometer (MZI) creating Gaussian-enveloped temporally modulated pulses at beat frequency $\Omega$, which is selected by varying the delay $\tau$. The temporally modulated pulses drive the optical rectification in DSTMS, generating narrow-band THz pulses at frequency $\Omega$. The generated THz fields are detected by EOS in a $200 \mu \mathrm{m}$ thick GaP crystal. WP: Wollaston prism, QWP: quarter-wave plate, and PM1-PM4: parabolic mirrors. (b) Pump spectrum (blue curve) after the MZI for $\tau=0.673$ corresponding to a beating frequency of $\Omega=1.8 \mathrm{THz}$. Reference spectrum (red dotted curve) in the absence of interference. The corresponding spectral modulation, calculated by removing the reference, is shown in the inset.
$E(t)=E_{0} \exp \left(-\alpha t^{2}\right) \exp \left(i \omega_{0} t+i b t^{2}\right)$, where $E_{0}$ is the electric field amplitude, $\omega_{0} /(2 \pi)$ is the laser carrier frequency, $b$ is the chirp rate, and $t_{c}=2 \sqrt{2 \ln 2 / \alpha}$ is the FWHM duration of the chirped pulse, which has been estimated to be approximately 1.55 ps from propagation simulation using the ZEMAX optical design code. The resulting temporal intensity from the superposition of these two time-shifted replica pulses by the MZI is: ${ }^{18}$

$$
\begin{aligned}
I(t, \tau)= & |E(t)+E(t+\tau)|^{2}=I_{1}(t)+I_{2}(t+\tau) \\
& +2 \sqrt{I_{1}(t) I_{2}(t+\tau)} \cos \left(\omega_{0} \tau+b \tau^{2}+2 b \tau t\right) .
\end{aligned}
$$

The time delay $\tau$ of the two pulses produces a Gaussian-enveloped, periodically modulated intensity with a beat frequency $\Omega=b \tau / \pi$, which is proportional to the chirp rate $b$. The temporally modulated pulse exhibits a comb-like structure in the frequency domain, where $\Omega$ corresponds to the comb spacing. The time delay $\tau$ between the two arms of the MZI can be changed to easily achieve the continuous tunability of the modulation frequency. Figure 1(b) shows the spectrum of the laser pulse measured by a fiber-coupled spectrometer (NIRquest OceanOptics, resolution $3.3 \mathrm{~nm}$ ). The beat frequency is $\Omega=1.8 \mathrm{THz}$ for $\tau=0.637 \mathrm{ps}$. The temporally modulated pulse generates narrow-band $\mathrm{THz}$ radiation by optical rectification in a $500 \pm 15 \mu$ m thick DSTMS (Rainbow Photonics). Because the chirped pulses are relatively long $(\sim 1.5 \mathrm{ps})$, the optical rectification from the individual Gaussian envelope is suppressed. This is confirmed by the negligible $\mathrm{THz}$ emission when the two replicas have no temporal overlap. The optical rectification becomes efficient when the beat frequency $\Omega$ is within the THz emission bandwidth of the DSTMS. Behind the $\mathrm{THz}$ generation, three low-pass filters (two at $20 \mathrm{THz}$ and one at $10 \mathrm{THz}$, made by QMC Instruments) are used to block the residual NIR beam (the overall extinction ratio for the pump exceeds $10^{5}$ ).

To reach intense fields, the $\mathrm{THz}$ beam is focused tightly by means of three off-axis parabolic mirrors (focal lengths of 1,6 , and 3 in.), achieving the spot size of around 1.5 times the diffraction limit. The temporal $\mathrm{THz}$ field is measured by electro-optic sampling (EOS) in $200 \mu \mathrm{m}$-thick GaP crystals using a $50 \mathrm{fs}, 800 \mathrm{~nm} 45 \mu \mathrm{m}$ low energy gating pulse obtained from the front-end Ti:sapphire laser.

Figure 2(a) shows the multi-cycle $\mathrm{THz}$ electric field generated by the modulated NIR pulse of Fig. 1(b). The spectral intensity, in Fig. 2(b), shows a Gaussian-like spectrum with its centroid at the beat frequency $\Omega=1.8 \mathrm{THz}$ and a FWHM bandwidth of $0.47 \mathrm{THz}$.
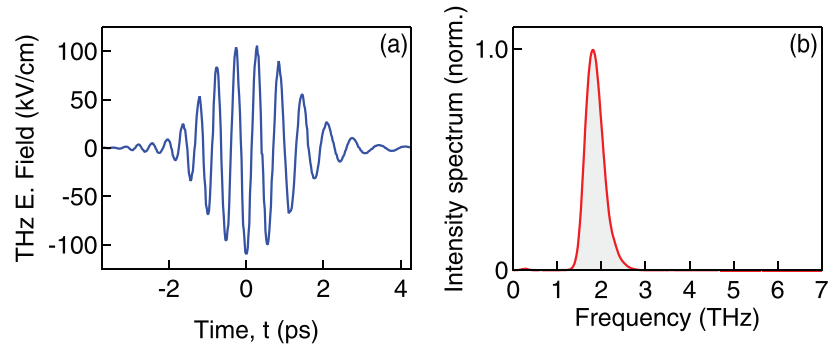

FIG. 2. (a) Electric field of the multi-cycle THz pulse generated for $\tau=0.637$ ps by optical rectification in $500 \mu \mathrm{m}$ thick DSTMS from the temporally modulated beam with the spectrum reported in Fig. 1(b). (b) Corresponding power spectrum. The $\mathrm{THz}$ emission by optical rectification is centered at the beat frequency $\Omega=1.8 \mathrm{THz}$. 
As observed in Fig. 2(a), the THz pulse shows a linear frequency chirp, which is due to the undesired third-order dispersion (TOD) introduced by the transmission gratings (see the supplementary material for the experimental evaluation and the analytical description).

The peak electric field $E_{\text {peak }}=\sqrt{\left.z_{0} E_{p} 4 \sqrt{\ln 2} /(\pi \sqrt{\pi} w)^{2} \tau_{F W H M}\right)}$ $=110 \pm 5 \mathrm{kV} / \mathrm{cm}$ was evaluated experimentally ${ }^{19}$ by measuring (i) the $\mathrm{THz}$ pulse energy, $E_{p}=0.15 \pm 0.01 \mu \mathrm{J}$, with a calibrated Golay cell; (ii) the waist size $w=390 \mu \mathrm{m}$, with a micro-bolometric $\mathrm{THz}$ camera (NEC IRV-T0830); (iii) the FWHM pulse duration, $t_{F W H M}^{T H z}=1.85 \mathrm{ps}$, from the EOS time-trace. The stability of the source against thermal and mechanical drifts over time is confirmed by repeatable EOS traces and modulated laser spectrum at the MZI output.

By changing the delay $\tau$, the $\mathrm{THz}$ center frequency can be continuously tuned over the emission band of the DSTMS, as shown in Fig. 3. Both the number of temporal oscillations and the $\mathrm{THz}$ carrier frequency increase with $\tau$ [Fig. 3(a)]. The corresponding spectra, shown in Fig. 3(b), indicate that the carrier frequency can be adjusted continuously between 0.5 and $6.5 \mathrm{THz}$ (i.e., over 3.7 octaves). The $\mathrm{THz}$ center frequency depends linearly on $\tau$, as shown in Fig. 3(c), with a slope of $3.3 \mathrm{THz} / \mathrm{ps}$. Because the $\mathrm{THz}$ center frequency corresponds to the beat frequency, $\Omega=b \tau / \pi$, we can estimate a chirp rate of $|b|=10.3 \mathrm{THz} / \mathrm{ps}$. The corresponding FWHM pulse duration of the chirped NIR pulse ${ }^{20}$ can be estimated using the approximated expression $t_{c}=\Delta \omega /(2 b)=2.1 \mathrm{ps}$ (with $\Delta \omega=2 \pi \cdot 7 \mathrm{THz}$ the measured FWHM spectral bandwidth), which agrees qualitatively with the values 1.5 ps calculated from the ZEMAX simulation. The bandwidth of
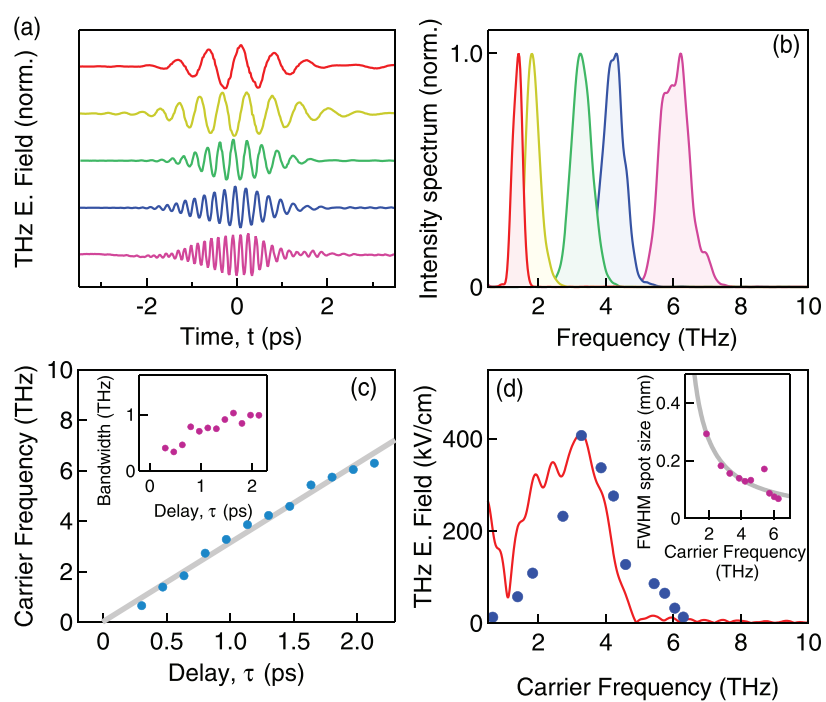

FIG. 3. (a) Multi-cycle THz waveforms and (b) corresponding spectra obtained by tuning the delay $\tau$. (c) Center frequency of the THz pulse shown as a function of $\tau$. The gray solid line is a linear fit with a slope of $3.3 \mathrm{THz} / \mathrm{ps}$. The inset shows the bandwidth of the narrow-band $\mathrm{THz}$ pulses. (d) Peak field of the multi-cycle $\mathrm{THz}$ pulses as a function of the center frequency (blue dots) compared to the spectral amplitude of the broadband $\mathrm{THz}$ pulse generated with a $60 \mathrm{fs}$ NIR pulse in the same crystal (red curve). Inset: FWHM spot size of the $\mathrm{THz}$ beam at focus (red dots), which scales with the inverse of $\mathrm{THz}$ carrier-frequency. The gray solid line is the corresponding fit. the THz pulses generated at various $\tau$, shown in the inset of Fig. 3(c), is lower than $0.5 \mathrm{THz}$ for delays $\tau<0.6 \mathrm{ps}$, which corresponds to a center frequency below $2 \mathrm{THz}$. The smaller bandwidth at lower $\tau$ may be ascribed to the strong absorption in DSTMS around $1 \mathrm{THz}$. The bandwidth and the number of cycles of the $\mathrm{THz}$ radiation can be controlled by the chirp-rate changing of the grating spacing. For the present measurements, the grating spacing is kept constant. However, the presence of the $\mathrm{THz}$ frequency chirp introduced by the TOD (see the supplementary material) prevents the generation of ultra narrow-band $\mathrm{THz}$ pulses due to the spectro-temporal dispersion of the carrier frequency. To achieve narrower and unchirped $\mathrm{THz}$ generation, the laser temporal modulation can be implemented by grating stretcher, ${ }^{21}$ which allows us to directly control the laser temporal shape by optical masks or spatial light modulators in the Fourier plane. The highest $\mathrm{THz}$ electric field strength $(410 \mathrm{kV} / \mathrm{cm})$ is achieved at a frequency around $3.8 \mathrm{THz}$ [Fig. 3(d)]. For this frequency, the measured $\mathrm{THz}$ energy is $0.5 \mu \mathrm{J}$. As expected, the frequency-scaling reproduces the spectral features of $\mathrm{THz}$ generated by a transform-limited pulse, as shown by the red curve in Fig. 1(d). The inset of Fig. 3(d) shows the focal spot size at different $\mathrm{THz}$ carrier frequencies measured by the $\mathrm{THz}$ camera: the spot size scales as inverse of the $\mathrm{THz}$ carrier frequency. In Table I, we report the main parameters of the present narrow-band and tunable $\mathrm{THz}$ source compared to previous similar setups.

Our narrow-band $\mathrm{THz}$ source is well suited for the selective control of low-energy collective modes. In order to demonstrate its versatility, we perform a resonant coherent phonon spectroscopy experiment on single crystal $\mathrm{SrCu}_{2}\left(\mathrm{BO}_{3}\right)_{2}$. This material has attracted considerable attention in the field of quantum magnetism, ${ }^{22}$ and for our purpose, it has very sharp phonon modes at low temperature, which lie in the spectral range of our $\mathrm{THz}$ source. The sample, grown using the floating zone method, is $120 \mu \mathrm{m}$ thick in the c direction and has an area of $\sim 4 \mathrm{~mm}^{2}$ in the a "b" plane $\mathrm{e}^{23}$ [Fig. 4(a)]. The narrowband $\mathrm{THz}$ pump pulse propagates along the c-axis with the electric field on the a "b" plane. In this configuration, $\mathrm{SrCu}_{2}\left(\mathrm{BO}_{3}\right)_{2}$ shows 3 non-fully symmetric $E$-modes ${ }^{24}$ located in our THz pump frequency region: $\nu_{1}=3.8 \mathrm{THz}, \nu_{2}=4.3 \mathrm{THz}$, and $\nu_{3}=4.6 \mathrm{THz}$. Because the $E$ symmetric modes are both IR-active, with the dipole moment in the a "b" plane, and Raman-active, they allow for a scheme of direct excitation at the $\Gamma$-point with $\mathrm{THz}$ pulse and femtosecond optical detection. ${ }^{25,26}$ By measuring the anisotropic transmission changes, $\Delta T / T$, at different delays with respect to the narrow-band $\mathrm{THz}$ pump, we detect the changes in susceptibility associated with the coherent atomic displacements related to $E$-modes. Similar to the EOS setup [Fig. 1(a)], the detected anisotropic transmission change, $\Delta T / T=\left(T_{1}-T_{2}\right) / T$, is proportional to the orthogonal polarization intensities of the probe, $I_{1}$ and $I_{2}$, shown in Fig. 4(a). These are separated by the Wollaston prism and detected by balanced photodiodes. $T$ is the probe transmittance without the THz pump.

The temporal evolution of the measured $\Delta T / T$ is depicted in Fig. 4(b). When the carrier frequency of the $\mathrm{THz}$ pump is tuned around $4 \mathrm{THz}$, we observe large-amplitude coherent oscillations from resonantly driven phonon modes. Because at low temperature $\mathrm{SrCu}_{2}\left(\mathrm{BO}_{3}\right)_{2}$ is a Mott insulator and the pump photon energy is far below the optical gap $\Delta_{0}=0.7 \mathrm{eV},{ }^{24}$ step-like functions or decay features related to excitation and relaxation of the electronic systems are not observed in the dynamics. Figure 4 (c) shows that the spectral 
TABLE I. Parameters of the intense narrow-band and tunable laser-based terahertz sources.

Method

Tuning range $(\mathrm{THz}) \quad$ Bandwidth $(\mathrm{THz}) \quad \mathrm{Max} \mathrm{THz}$ energy $(\mu \mathrm{J}) \quad$ Max strength $(\mathrm{MV} / \mathrm{cm}) \quad$ Nonlinear crystal

\begin{tabular}{|c|c|c|c|c|c|}
\hline Chirp + MZI (this work) & $0.5-6.5$ & $<0.5$ & 0.5 & 0.4 & DSTMS \\
\hline MZI + compressor ${ }^{15}$ & $0.25-3$ & $<0.25$ & 6.7 & 2.3 & $\mathrm{OH} 1$ \\
\hline $\mathrm{MZI}+$ compressor $(10 \mathrm{~Hz})^{16}$ & $0.7-7$ & $<0.5$ & 20 & 8 & DSTMS \\
\hline Chirp + ethalon ${ }^{13}$ & $0.3-1.3$ & $<0.1$ & 1.5 & $\ldots$ & $\mathrm{LiNbO}_{3}$ \\
\hline Chirp+ethalon ${ }^{14}$ & $0.3-0.8$ & $<0.1$ & 0.08 & $\ldots$ & OHQ-TMS \\
\hline $\mathrm{DFG}^{11}$ & $4-18$ & $<1$ & 1.9 & 3.7 & DSTMS \\
\hline
\end{tabular}

amplitude of the measured $\Delta T / T$ displays strong and narrow peaks at $3.8 \mathrm{THz}$ and $4.6 \mathrm{THz}$.

To understand the observed dynamics, we model the coherent lattice displacement as a sum of damped harmonic oscillators driven by the $\mathrm{THz}$ electric field of the pump, as shown by the dotted black curve in Fig. 4(c). The equation of motion for normal coordinate $Q_{i}(t)$ along the displacement vector of the $i$ th phonon mode is ${ }^{25}$

$$
\frac{d^{2} Q_{i}(t)}{d t^{2}}+2 \gamma_{i} \frac{d Q_{i}(t)}{d t}+\omega_{i}^{2} Q_{i}(t)=\frac{e_{i}^{*}}{m_{i}^{*}} E_{T H z}^{e f f}(t),
$$

where $\omega_{i}=2 \pi \nu_{i}$ and $\gamma_{i}$ are the phonon angular eigenfrequencies and the damping rate, while $e_{i}^{*}$ and $m_{i}^{*}$ are the effective charge and the reduced mass of the mode. The effective driving electric field $E_{T H z}^{\text {eff }}(t)$ inside the sample corresponds to $E_{T H z}^{e f f}(\nu)=t(\nu) E_{T H z}(\nu)$ in the frequency domain, where $E_{T H z}(\nu)$ is the Fourier transform of the pump electric field, $t(\nu)=2 /(1+\tilde{n}(\nu))$ is the THz transmission amplitude, and $\tilde{n}(\nu)$ is the complex refractive index, which is strongly frequencydependent at the IR-active lattice resonances. The refractive index is derived from the complex dielectric function ${ }^{24} \tilde{\epsilon}=\epsilon_{\infty}+\sum_{i} \omega_{p, i}^{2} /$ $\left(\omega_{i}^{2}-\omega^{2}-i \gamma_{i} \omega\right)$, where $\omega_{p, i}$ is the plasma frequency and $\gamma_{i}$ the damping rate. The anisotropic transmission change associated with the atomic coordinate $Q_{i}(t)$ is connected to the Raman tensor $R_{k j}^{i}$ of
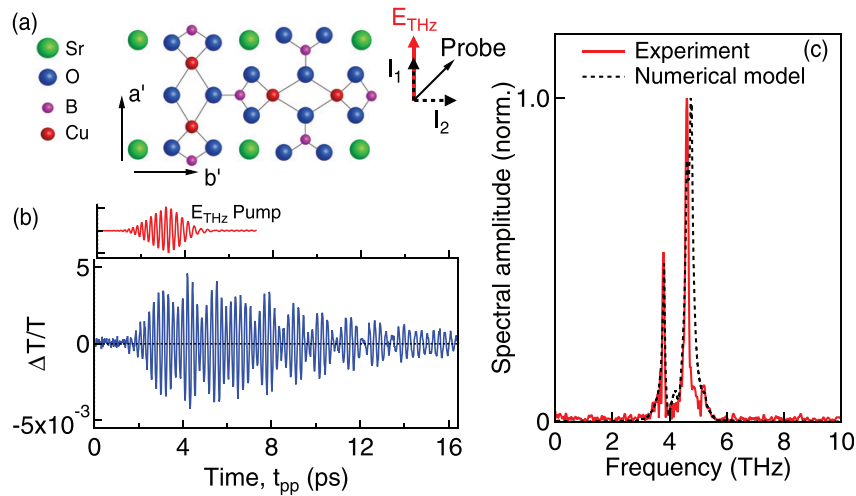

FIG. 4. (a) Lattice structure of $\mathrm{SrCu}_{2}\left(\mathrm{BO}_{3}\right)_{2}$ in the $\mathrm{a}^{\prime} \mathrm{b}^{\prime}$ plane and experimental geometry. The $\mathrm{THz}$ pump electric field is polarized along the vertical direction. (b) Evolution of anisotropic transmission changes $\Delta T / T$ caused by $\mathrm{THz}$ excitation of the E-symmetric phonon modes. (c) Spectral amplitude obtained by Fourier transformation (red curve) and from the model of Lorentz oscillators given in Eq. (2) (black dotted curve). The sample temperature is $4 \mathrm{~K}$. the $i$ th $E$-symmetry mode by $\Delta T / T \propto R_{k j}^{i} Q_{i}$. By using the parameters for the three phonon modes taken from Ref. 24, $\omega_{p, 1}=125 \mathrm{~cm}^{1}$ $(3.8 \mathrm{THz}), \quad \omega_{p, 2}=141 \mathrm{~cm}^{1} \quad(4.3 \mathrm{THz}), \omega_{p, 3}=157 \mathrm{~cm}^{1} \quad(4.7 \mathrm{THz})$, $\gamma_{1}=1.0 \mathrm{~cm}^{1}, \gamma_{2}=1.8 \mathrm{~cm}^{1}$, and $\gamma_{3}=0.7$, we calculate the transmission changes in the frequency domain by using Eq. (2) and the driving THz field measured by EOS. The only free parameters are the relative magnitudes of the peaks $\left(A_{E_{1}}, A_{E_{2}}, A_{E_{2}}\right)$, which are linearly proportional to the dipole coupling terms $e_{i}^{*} / m_{i}^{*}$ and to the amplitude of the Raman tensors $R_{k j}$ of the modes. As shown in Fig. 4(c), the numerical calculation reproduces the experimental data and captures the relative peak ratios $A_{E_{2}} / A_{E_{1}}=0.3$ and $A_{E_{3}} / A_{E_{1}}=4$. This is expected from Raman spectroscopy because the intensities of $E_{1}$ and $E_{2}$ are larger than those of $E_{2}$ at room temperature ${ }^{27}$ and conceivably also at low temperature. In the calculation, we assumed an equal coupling term $e_{i}^{*} / m_{i}^{*}$ for the phonon modes because they have a comparable net dipole moment. ${ }^{24}$ In Fig. 5, we show the THz-driven dynamics of $\Delta T / T$ as a function of

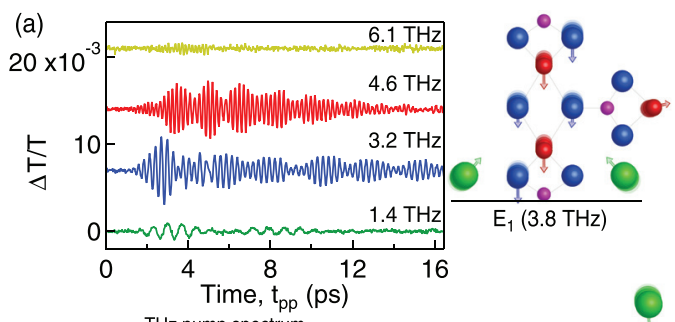

(c)
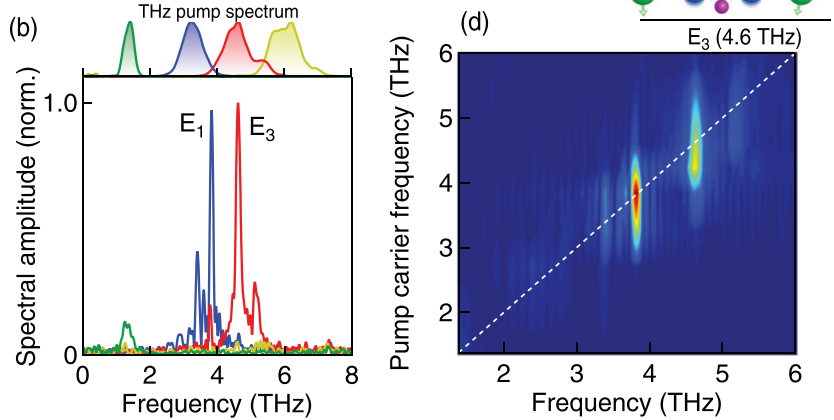

FIG. 5. (a) Dynamics of $\Delta T / T$ at different $\mathrm{THz}$ pump carrier frequencies: $1.4 \mathrm{THz}$, 3.2 THz, 4.6 THz, and 6.1 THz. (b) Corresponding spectral amplitude $\Delta T / T(\nu)$ for $t_{p p}>4.2 \mathrm{ps}$ (when $E_{T H z}=0$ ) and related THz pump spectra (top panel). (c) Atomic displacement vectors projected in the $a \mathrm{~b}$ plane for the observed infraredactive $E_{1}$ and $E_{3}$ phonon modes, from Ref. 24. (d) Normalized amplitude $\Delta T / T(\nu)$ for $t_{p p}>4.2 \mathrm{ps}$ as a function of the carrier frequency of the THz pump. The $45^{\circ}$ dashed line is used as a guide to the eye. Red and blue colors correspond to $\Delta T / T(\nu)=1$ and $\Delta T / T(\nu)=0$, respectively. 
the pump carrier frequency. In particular, Fig. 5(a) displays the pumpprobe time-traces for pump frequencies at $1.4 \mathrm{THz}, 3.2 \mathrm{THz}, 4.6 \mathrm{THz}$, and 6.1 THz. The corresponding Fourier transforms are shown in Fig. 5(b). For the off-resonant THz pump frequencies at $1.4 \mathrm{THz}$ and $6.1 \mathrm{THz}$, no long-lived coherent oscillations are observed in the dynamics of $\Delta T / T$, while for pumping at $3.8 \mathrm{THz}$ and $4.6 \mathrm{THz}$, the $\mathrm{E}_{1}$ mode and $\mathrm{E}_{3}$ resonances are excited selectively. The weak oscillations for the off-resonant pump are ascribed to the electro-optic response of $\mathrm{SrCu}_{2}\left(\mathrm{BO}_{3}\right)_{2}$. It is evident that the $\mathrm{THz}$ pump can target different atomic displacements associated with the $E_{1}$ and $E_{3}$ phonon modes, which are depicted in Fig. 5(c). In Fig. 5(d), we present the $2 \mathrm{D}$ map of the amplitude spectrum of $\Delta T / T$ as a function of the $\mathrm{THz}$ carrier frequency of the pump.

In summary, we generate intense and tunable narrow-band $\mathrm{THz}$ pulses by optical rectification in DSTMS of a modulated laser intensity profile. This source allows subterahertz linewidth and carrierfrequency tunability from 0.5 to $6.5 \mathrm{THz}$, which is not available with other high repetition-rate sources based on standard lasers. These characteristics are highly desirable for the selective excitation of IRactive modes and, in general, for advanced time-resolved spectroscopy. By using a coherent phonon detection scheme, we demonstrated that the narrow-band $\mathrm{THz}$ source can resonantly drive large-amplitude lattice displacements associated with non-fully symmetric modes in single-crystal $\mathrm{SrCu}_{2}\left(\mathrm{BO}_{3}\right)_{2}$. We found that the excited phonon spectrum is well reproduced by a model of Lorentz oscillators driven by multi-cycle narrow-band $\mathrm{THz}$ pulses. By tuning the carrier frequency of the THz stimulus, we can select a single phonon mode to drive. The compact source we present based on commercially available OPA is up-scalable to higher $\mathrm{THz}$ fields, offering viable approaches for the study of phonon dependent nonlinearities in condensed matter.

See the supplementary material for the experimental evaluation and the analytical description of the $\mathrm{THz}$ chirp.

The authors thank E. J. Divall, B. Normand, and B. Wehinger for their support. This research was supported by the European Research Council (ERC) under EU Horizon 2020 Research and Innovation Program Grant No. 681654 (HyperQC).

\section{DATA AVAILABILITY}

The data that support the findings of this study are available from the corresponding author upon reasonable request.

\section{REFERENCES}

${ }^{1}$ M. Mitrano, A. Cantaluppi, D. Nicoletti, S. Kaiser, A. Perucchi, S. Lupi, P. D. Pietro, D. Pontiroli, M. Riccò, S. R. Clark, D. Jaksch, and A. Cavalleri, "Possible light-induced superconductivity in $\mathrm{K}_{3} \mathrm{C}_{60}$ at high temperature," Nature 530, 461-464 (2016).

${ }^{2}$ T. F. Nova, A. Cartella, A. Cantaluppi, M. Först, D. Bossini, R. V. Mikhaylovskiy, A. V. Kimel, R. Merlin, and A. Cavalleri, "An effective magnetic field from optically driven phonons," Nat. Phys. 13, 132-136 (2017).

${ }^{3}$ R. Mankowsky, A. Subedi, M. Först, S. O. Mariager, M. Chollet, H. T. Lemke, J. S. Robinson, J. M. Glownia, M. P. Minitti, A. Frano, M. Fechner, N. A. Spaldin, T. Loew, B. Keimer, A. Georges, and A. Cavalleri, "Nonlinear lattice dynamics as a basis for enhanced superconductivity in $\mathrm{YBa}_{2} \mathrm{Cu}_{3} \mathrm{O}_{6.5}$," Nature 516, 71-73 (2014).

${ }^{4}$ M. Först, C. Manzoni, S. Kaiser, Y. Tomioka, Y. Tokura, R. Merlin, and A. Cavalleri, "Nonlinear phononics as an ultrafast route to lattice control," Nat. Phys. 7, 854-856 (2011).

${ }^{5}$ R. Merlin, "Generating coherent THz phonons with light pulses," Solid State Commun. 102, 207-220 (1997).
${ }^{6}$ H. J. Zeiger, J. Vidal, T. K. Cheng, E. P. Ippen, G. Dresselhaus, and M. S. Dresselhaus, "Theory for displacive excitation of coherent phonons," Phys. Rev. B 45, 768-778 (1992).

${ }^{7}$ A. Melnikov, O. Misochko, and S. Chekalin, "Generation of coherent phonons in bismuth by ultrashort laser pulses in the visible and NIR: Displacive versus impulsive excitation mechanism," Phys. Lett. A 375, 2017-2022 (2011).

${ }^{8}$ P. Salén, M. Basini, S. Bonetti, J. Hebling, M. Krasilnikov, A. Y. Nikitin, G. Shamuilov, Z. Tibai, V. Zhaunerchyk, and V. Goryashko, "Matter manipulation with extreme terahertz light: Progress in the enabling THz technology," Phys. Rep. 836-837, 1-74 (2019).

${ }^{9} \mathrm{D}$. Nicoletti and A. Cavalleri, "Nonlinear light-matter interaction at terahertz frequencies," Adv. Opt. Photonics 8, 401 (2016).

${ }^{10} \mathrm{M}$. Jazbinsek, U. Puc, A. Abina, and A. Zidansek, "Organic crystals for THz photonics," Appl. Sci. 9, 882 (2019).

${ }^{11}$ B. Liu, H. Bromberger, A. Cartella, T. Gebert, M. Först, and A. Cavalleri, "Generation of narrowband, high-intensity, carrier-envelope phase-stable pulses tunable between 4 and 18 THz,” Opt. Lett. 42, 129 (2017).

${ }^{12}$ K.-L. Yeh, J. Hebling, M. C. Hoffmann, and K. A. Nelson, "Generation of high average power $1 \mathrm{kHz}$ shaped $\mathrm{THz}$ pulses via optical rectification," Opt. Commun. 281, 3567-3570 (2008).

${ }^{13}$ Z. Chen, X. Zhou, C. A. Werley, and K. A. Nelson, "Generation of high power tunable multicycle teraherz pulses,” Appl. Phys. Lett. 99, 071102 (2011).

${ }^{14}$ J. Lu, H. Y. Hwang, X. Li, S.-H. Lee, O.-P. Kwon, and K. A. Nelson, "Tunable multicycle THz generation in organic crystal HMQ-TMS," Opt. Express 23, 22723 (2015).

${ }^{15}$ O. V. Chefonov, A. V. Ovchinnikov, C. P. Hauri, and M. B. Agranat, "Broadband and narrowband laser-based terahertz source and its application for resonant and non-resonant excitation of antiferromagnetic modes in $\mathrm{NiO}$," Opt. Express 27, 27273 (2019).

${ }^{16}$ C. Vicario, A. V. Ovchinnikov, O. V. Chefonov, and C. P. Hauri, "Multi-octave spectrally tunable strong-field terahertz laser," arXiv:1608.05319v1 (2016).

${ }^{17}$ A. Trisorio, P. M. Paul, F. Ple, C. Ruchert, C. Vicario, and C. P. Hauri, "Ultrabroadband TW-class Ti: Sapphire laser system with adjustable central wavelength, bandwidth and multi-color operation," Opt. Express 19, 20128 (2011).

${ }^{18}$ Y. Shen, X. Yang, G. L. Carr, Y. Hidaka, J. B. Murphy, and X. Wang, "Tunable few-cycle and multicycle coherent terahertz radiation from relativistic electrons," Phys. Rev. Lett. 107, 204801 (2011).

${ }^{19}$ H. Roskos, M. Thomson, M. Kreß, and T. Löffler, "Broadband THz emission from gas plasmas induced by femtosecond optical pulses: From fundamentals to applications," Laser Photonics Rev. 1, 349-368 (2007).

${ }^{20}$ W. Fan, B. Zhu, Y. Wu, F. Qian, M. Shui, S. Du, B. Zhang, Y. Wu, J. Xin, Z. Zhao, L. Cao, Y. Wang, and Y. Gu, "Measurement of the chirp characteristics of linearly chirped pulses by a frequency domain interference method," Opt. Express 21, 13062 (2013).

${ }^{21} \mathrm{D}$. Goswami, "Optical pulse shaping approaches to coherent control," Phys. Rep. 374, 385-481 (2003).

${ }^{22}$ M. E. Zayed, C. Rüegg, J. Larrea, Jr., A. M. Läuchli, C. Panagopoulos, S. S. Saxena, M. Ellerby, D. F. McMorrow, T. Strässle, S. Klotz, G. Hamel, R. A. Sadykov, V. Pomjakushin, M. Boehm, M. Jiménez-Ruiz, A. Schneidewind, E. Pomjakushina, M. Stingaciu, K. Conder, and H. M. Rønnow, "4-spin plaquette singlet state in the Shastry-Sutherland compound $\mathrm{SrCu}_{2}\left(\mathrm{BO}_{3}\right)_{2}$," Nat. Phys. 13, 962-966 (2017).

${ }^{23}$ K. Kakurai, K. Nukui, N. Aso, M. Nishi, H. Kadowaki, H. Kageyama, Y. Ueda, L.-P. Regnault, and O. Cépas, "Neutron scattering investigation on quantum spin system $\mathrm{SrCu}_{2}\left(\mathrm{BO}_{3}\right)_{2}$," Prog. Theor. Phys. Suppl. 159, 22-32 (2005).

${ }^{24}$ C. C. Homes, S. V. Dordevic, A. Gozar, G. Blumberg, T. Rõõm, D. Hüvonen, U. Nagel, A. D. LaForge, D. N. Basov, and H. Kageyama, "Infrared spectra of the low-dimensional quantum magnet $\mathrm{SrCu}_{2}\left(\mathrm{BO}_{3}\right)_{2}$ : Measurements and abinitio calculations," Phys. Rev. B 79, 125101 (2009).

${ }^{25}$ T. Huber, M. Ranke, A. Ferrer, L. Huber, and S. L. Johnson, "Coherent phonon spectroscopy of non-fully symmetric modes using resonant terahertz excitation,” Appl. Phys. Lett. 107, 091107 (2015).

${ }^{26}$ A. A. Melnikov, K. N. Boldyrev, Y. G. Selivanov, V. P. Martovitskii, S. V. Chekalin, and E. A. Ryabov, "Coherent phonons in a bi ${ }_{2} \mathrm{se}_{3}$ film generated by an intense single-cycle THz pulse,” Phys. Rev. B 97, 214304 (2018).

${ }^{27}$ K.-Y. Choi, Y. G. Pashkevich, K. V. Lamonova, H. Kageyama, Y. Ueda, and P. Lemmens, "Strong anharmonicity and spin-phonon coupling in the quasi-two-dimensional quantum spin system $\mathrm{Sr}_{1-x} \mathrm{Ba}_{\mathrm{x}} \mathrm{Cu}_{2}\left(\mathrm{BO}_{3}\right)_{2}$," Phys. Rev. B 68, 104418 (2003). 